\title{
Voltage Control of Distribution Networks with Distributed Generation using Reactive Power Compensation
}

\author{
M. A. Mahmud, Student Member, IEEE, M. J. Hossain, Member, IEEE, H. R. Pota, and A. B. M. Nasiruzzaman
}

\begin{abstract}
Voltage profile of distribution networks with distributed generation are affected significantly due to the integration of distributed generation (DG) on it. This paper presents a way to control voltage of distribution networks with DG using reactive power compensation approach. In this paper, the voltage control approach is shown based on the worst case scenario of the network. To keep the voltage profile within the specified limits, it is essential to regulate the reactive power of the compensators. Finally, based on this concept, a static analysis is done on a 15- bus Japanese distribution system, called Kumumoto system where the system is modified with the inclusion of distributed wind generators, photovoltaics, and synchronous generators.
\end{abstract}

\section{INTRODUCTION}

Penetration of DG into distribution network is entering an emerging era of rapid expansion and commercialization. Traditionally, the distribution networks are passive networks where the flow of both real and reactive power is always from the higher to lower voltage levels. However, with significant penetration of distributed generation, the power flows may become reversed since the distribution network is no longer a passive circuit supplying loads but an active system with power flows and voltages determined by the generation as well as load. Hence, there are dramatic changes in the nature of distribution networks with distributed generation.

The changes within the distribution networks due to the integration of DG create some provisions which have positive as well as negative impacts to both distribution network service providers (DNSPs) and customers. Some technical benefits of DG in terms of voltage profile improvement, line-loss reduction, and environmental impact reduction are proposed in [1]. A multi objective performance index for distribution networks with distributed generation is proposed in [2], which considers a wide range of technical issues. The study proposed in [3] for DG planning models, consider the various technical requirements such as thermal ratings of the equipments, voltage rise, systems fault levels, reverse power flow capability of the tap-changers, power losses, power quality (such as flicker, harmonics), protection, etc. A time-series based maximization

M. A. Mahmud, H. R. Pota, and A. B. M. Nasiruzzaman are with the School of Engineering and Information Technology (SEIT), The University of New South Wales at Australian Defence Force Academy (UNSW@ADFA), Canberra, ACT 2600, Australia. E-mail: Md.Mahmud@student.adfa.edu.au, h.pota@adfa.edu.au, and nasiruzzaman@ieee.org

M. J. Hossain is with the School of Information Technology and Electrical Engineering (ITEE), University of Queensland, St Lucia, Brisbane, QLD 4072, Australia. E-mail: m.hossain9@uq.edu.au. method is described in [4], to maximize the distributed wind power generation.

Maximization of distributed generation into conventional electric distribution networks reduces power loss, is not always true. If the distributed generators are not placed properly, power loss may increase which is shown in [5]. In [6], a method is proposed to identify the optimal bus for placing DG in a networked system based on bus admittance matrix, generation information, and load distribution of the system. An analytical approach to calculate optimal size and location of DG has been discussed in [7]. The limitation of this proposed technique is that the loss sensitivity factor may not lead to the best placement for loss reduction.

Integration of distributed generation in distribution systems is susceptible to voltage variation. Moreover, the impact of losing a single or a few distributed generators following a remote fault may not be a significant issue, but the connection or disconnection of a large fraction of distributed generation units may become problematic which may lead to sudden appearance of hidden loads and affect the voltage profile of low voltage distribution network. To overcome these problems, the distribution network capacity is determined for distributed generation by using optimal power flow with voltage step constraints in [8]. The voltage rise issues due to the distributed generation is discussed nicely by giving some theoretical background in [3]. But the detailed analysis of voltage rise effects is not discussed in [3]. Also there is no indication in [3], about how much DG can be connected to the distribution networks. A multi-period AC optimal power flow (OPF)-based technique is proposed in [9], for evaluating the maximum capacity of new variable distributed generation which can be connected to a distribution network when active network management (ANM) control strategies are in place. Recently, the worst case scenario of the distribution systems with DG is considered in our work [10], [11] which is useful for both the customers and DNSPs. But the considerations of voltage control are out of scope of these papers [1-11].

The maximum permissible limits of voltage variation depend on the policies and regulation of a country which may be different for different countries. A stochastic evaluation approach of voltage control is proposed in [12], for maintaining the voltages within the accepted limits. The main limitation of the stochastic evaluation method proposed in [12] is that it needs more computation efforts when the voltages are beyond the accepted limits. A fuzzy-based reactive power and voltage control approach is proposed in [13] and a nonlinear 


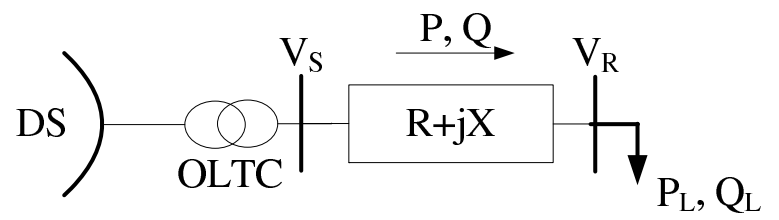

Fig. 1. Conventional Two-bus Distribution System

interior-point method and discretization penalties is proposed in [14] where the switching operation of load tap changers and capacitors are considered to minimize the voltage variation. But the accuracy of these approaches [13], [14] are not feasible. The more accuracies are obtained by the methods proposed in [15], [16]. The main problem of the method proposed in [16] is that the purpose is not to control the bus voltages rather to guarantee that active power generation does not cause voltage rise. On the other hand, an optimization approach is used in [15], to control the voltage at both transmission and distribution level by optimizing the power factor and tap-changer settings of the distribution networks to keep the voltages within the permissible limits which has no clarification about voltages of different buses.

The aim of this paper is to propose a voltage control approach for distribution network with DG by using reactive power compensation. To do this, first a voltage variation formula is derived and then the amount of reactive power compensation is determined based on the worst case scenario of the network. Finally, the proposed algorithm is implemented on a 15- bus Japanese system.

\section{Voltage VARiation in CONVENTIONAL DISTRIBUTION NETWORK}

Most of the distribution networks are modeled as passive networks with radial configuration and as mentioned in the literature, the flow of power both real $(P)$ and reactive $(Q)$, is always from the higher to lower voltage levels. The amount of voltage drop can be calculated from the analysis of two-bus distribution system as shown in Fig. 1.

In Fig. 1, DS and $O L T C$ stand for the distribution systems and on-load tap-changer respectively, $V_{S}$ is the sending end voltage, $V_{R}$ is the receiving end voltage, $P$ and $Q$ are the real and reactive power flowing through the distribution network to the customer, i.e., these are supplied from distribution substation (DS), and $P_{L}$ and $Q_{L}$ are the real and reactive power of the load. The voltage at the sending end can be written as

$$
\widehat{V}_{S}=\widehat{V}_{R}+\widehat{I}(R+j X)
$$

where $\widehat{I}(I=|\widehat{I}|)$ is the phasor representation of the current flowing through the line. The power supplied from the distribution system can be written as

$$
P+j Q=\widehat{V}_{S} \widehat{I}^{*}
$$

Therefore, the current flowing through the line can be written as

$$
\widehat{I}=\frac{P-j Q}{\widehat{V}_{S}}
$$

By using the value of $\widehat{I}$, the sending end voltage can be expressed as

$$
\begin{aligned}
\widehat{V}_{S} & =\widehat{V}_{R}+\frac{P-j Q}{\widehat{V}_{S}}(R+j X) \\
& =\widehat{V}_{R}+\frac{R P+X Q}{\widehat{V}_{S}}+j \frac{X P-R Q}{\widehat{V}_{S}}
\end{aligned}
$$

Therefore, the voltage drop between the sending end and recieving end can be written as

$$
\Delta \widehat{V}=\widehat{V}_{S}-\widehat{V}_{R}=\frac{R P+X Q}{\widehat{V}_{S}}+j \frac{X P-R Q}{\widehat{V}_{S}}
$$

Since the angle between the sending end voltage and the receiving end voltage is very small, the voltage drop is approximately equal to the real part of the drop [17] and if the sending end bus is considered as reference bus, the angle of this voltage is 0 , i.e., $\widehat{V_{S}}=\left|V_{S}\right|=V_{S}$. Therefore, the above equation can be approximated as

$$
\Delta V \approx \frac{R P+X Q}{V_{S}}
$$

If the sending end voltage of the system as shown in Fig. 1 is considered as the base voltage, then $V_{S}$ can be assumed as unity. Therefore, equation (1) can be written as follows:

$$
\Delta V \approx R P+X Q
$$

The amount of voltage variation in a large distribution network as shown Fig. 2 can be determined by using the same formula as shown by equation (1). In Fig. 2, an n-bus system is considered. The voltage drop between $i^{t h}$ and $j^{t h}$ bus can be written as

$$
\Delta V_{i j} \approx \frac{R_{i j} P_{i j}+X_{i j} Q_{i j}}{V_{i}}
$$

where, $\Delta V_{i j}$ is the variation of voltage between $i^{t h}$ and $j^{\text {th }}$ bus, $R_{i j}$ is the resistance between $i^{t h}$ and $j^{\text {th }}$ bus, $X_{i j}$ is the reactance between $i^{t h}$ and $j^{t h}$ bus, $V_{i}$ is the voltage at $i^{t h}$ bus, and $P_{i j}$ and $Q_{i j}$ are the active and reactive power flowing from $i^{\text {th }}$ to $j^{\text {th }}$ bus.

The voltage level at each connection point of the load is very important for the quality of the supply. Since there are no internationally agreed rules that define the allowed steadystate voltage range, the maximum permitted voltage variation on each bus-bar is defined by some technical regulations or specific contracts.

\section{Voltage VARiation in Distribution Network WITH DG}

When generators are connected to the distribution system, the power flow and the voltage profiles are affected as well as the system is no longer passive but active. In order to export power, a generator is likely to operate at a higher voltage as compared to the other nodes where it is supplying power. This can be explained by using equation (2). In this case, the receiving end voltage $\left(V_{R}\right)$ will be

$$
V_{R} \approx V_{S}+R P+X Q
$$




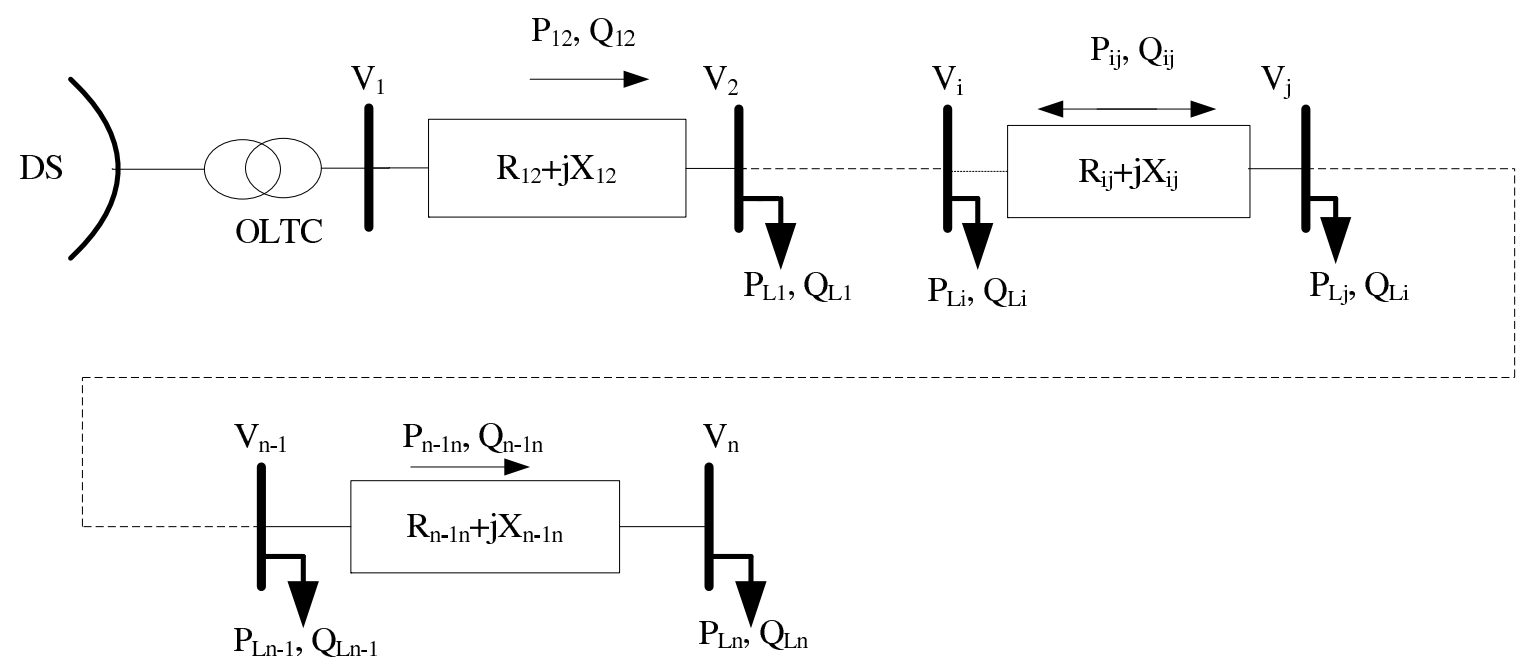

Fig. 2. Conventional n-bus Large Distribution System

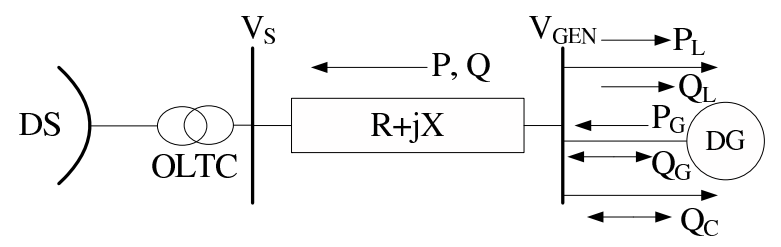

Fig. 3. Two-bus Distribution System with DG

as the direction of the power flow is reversed. Thus, the voltage at the point of connection of the generator will rise above the sending end voltage which can be clarified through Fig. 3 .

In Fig. 3, a distributed generator (DG) is connected where the voltage $\left(V_{G E N}\right)$ is assumed to be $11 \mathrm{kV}, P_{G}$ and $Q_{G}$ are the generated active and reactive power, respectively, by the DG, $P_{L}$ and $Q_{L}$ are the active and reactive power of the load respectively and $Q_{C}$ is reactive power of the shunt compensator. This DG with load and compensator is connected to the distribution system (DS) via overhead distribution line with impedance $R+j X$ and through OLTC. The voltage rise along the distribution network as shown in Fig. 3 can be written as follows:

$$
\Delta V=V_{G E N}-V_{S} \approx \frac{R P+X Q}{V_{G E N}}
$$

where, $P=\left(P_{G}-P_{L}\right), Q=\left( \pm Q_{C}-Q_{L} \pm Q_{G}\right)$. If $V_{G E N}$ is expressed in terms of per unit, then equation (4) can be written as

$$
\Delta V=V_{G E N}-V_{S} \approx R\left(P_{G}-P_{L}\right)+X\left( \pm Q_{C}-Q_{L} \pm Q_{G}\right)
$$

The generators always export active power $\left(+P_{G}\right)$ and may export or import reactive power $\left( \pm Q_{G}\right)$, whereas the load consumes both active $\left(-P_{L}\right)$ and reactive $\left(-Q_{L}\right)$ power and the compensators may export or absorb only reactive power $\left( \pm Q_{C}\right)$. Recently, small synchronous generators through combined heat power (CHP) generation scheme, small wind turbine, and photovoltaic (PV) are widely used as distributed generators. In CHP generation scheme, the synchronous generator exports real power even when the electrical load of the systems falls below the output of the generator but it may absorb or export reactive power depending on the setting of the excitation system of the generator. The wind turbine also exports real power but it absorbs reactive power as its induction generator requires a source of reactive power to operate. The photovoltaic (PV) systems are used to export real power at a set power factor but may introduce harmonic currents. Therefore, the power flows through the circuits may be in either direction depending on the relative magnitudes of the real and reactive network loads compared to the generator outputs and any losses in the network.

The voltage variation in the large distribution networks with distributed generation can be analyzed in a similar way to that of conventional distribution networks as mentioned in the previous section. If we integrate distributed generation at $j^{\text {th }}$ bus of the conventional distribution network as shown in Fig. 2, the system will be converted like Fig. 4.

The voltage variation $\Delta V_{j i}$ at the DG connection point, i.e., at the $j^{\text {th }}$ bus of a radial distribution feeder can be written as

$$
\Delta V_{j i} \approx \frac{R_{i j}\left(P_{G j}-P_{L j}\right)+X_{i j}\left( \pm Q_{G j}-Q_{L j}\right)}{V_{j}}
$$

where, $P_{G j}$ is the active power supplied by DG, $Q_{G j}$ is the reactive power supplied or absorbed by DG depending on the nature of DG as discussed before, $P_{L j}$ and $Q_{L j}$ are the active and reactive power of the load connected to the $j^{\text {th }}$ bus of the distribution system respectively. If we connect a shunt compensator at point of DG connection with reactive power $Q_{C j}$, the equation (7) can be written as

$$
\Delta V_{j i} \approx \frac{R_{i j}\left(P_{G j}-P_{L j}\right)+X_{i j}\left( \pm Q_{G j} \pm Q_{C j}-Q_{L j}\right)}{V_{j}}
$$

The variation of voltages in the small as well as large distribution network can be determined by using the formulae (4)-(8). 


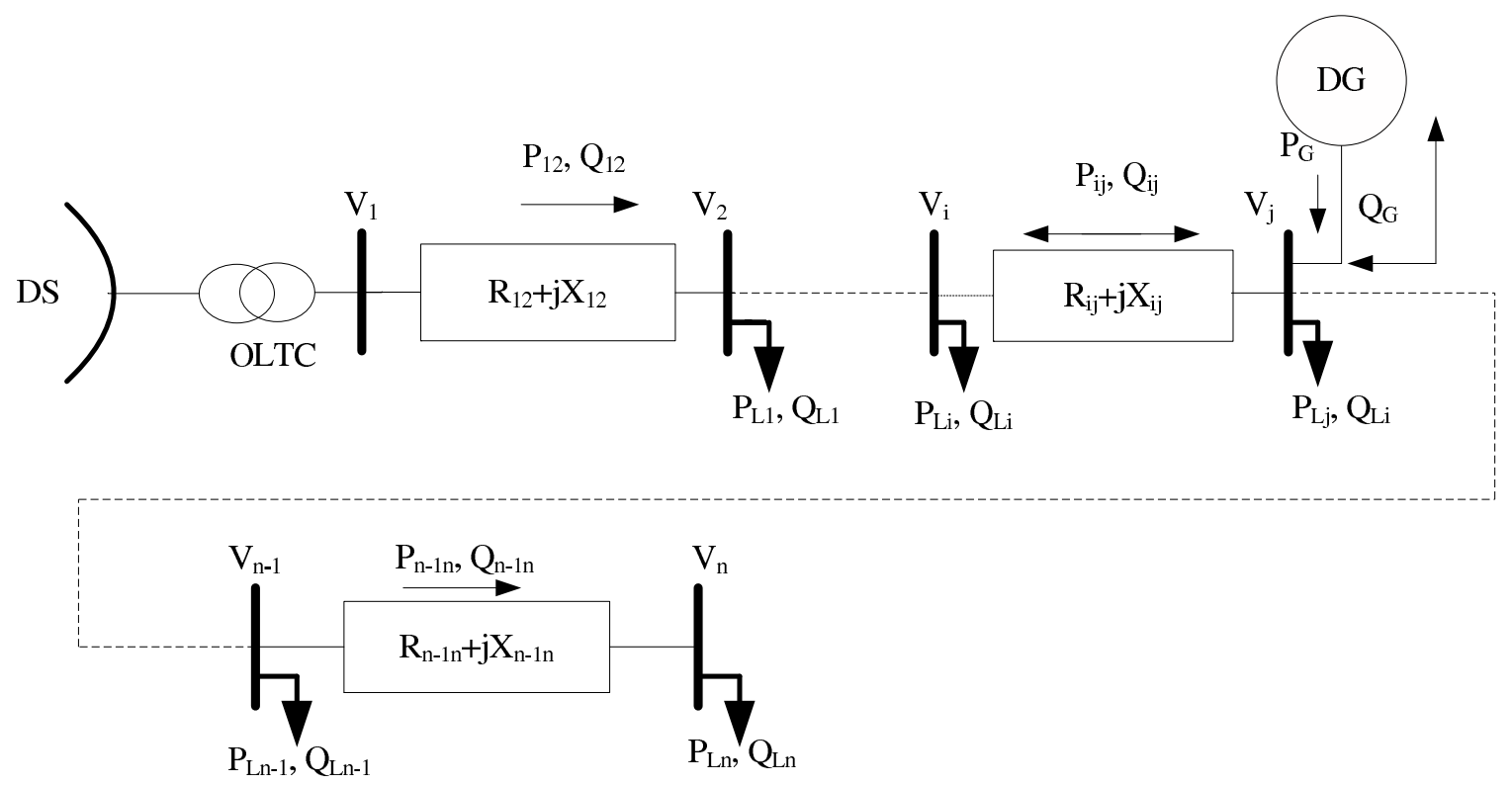

Fig. 4. n-bus Large Distribution System with Distributed Generation

\section{Worst Case Scenario of Distribution Network WITH DG}

The distributed generators are connected to the distribution system due to the technological innovations and change in economic and regulatory environment as well as to meet the increased load demand. From equation (6), we can write,

$$
P_{G} \approx \frac{V_{G E N}-V_{S}+R P_{L}-X\left( \pm Q_{C}-Q_{L} \pm Q_{G}\right)}{R}
$$

For large distribution network, the above equation can be written as

$$
P_{G j} \approx \frac{V_{j}-V_{i}+R_{i j} P_{L j}-X_{i j}\left( \pm Q_{C j}-Q_{L j} \pm Q_{G j}\right)}{R_{i j}}
$$

From equation (9), it is clear that the level of generation that can be connected to the distribution system depends on the following factors:

- voltage at the primary DES

- voltage level of the receiving end

- size of the conductors as well the distance from primary DES

- load demand on the system

- other generation on the system

When a generator is to be connected to the distribution system, the DNSP should consider the worst case operating scenarios to easily demonstrate the relationship between voltage rise and the DG connected to the DES and also they should ensure that their network and customer will not be adversely affected. Generally, these worst case scenarios are:

- minimum load maximum generation

- maximum load minimum generation

- maximum load maximum generation

The amount of voltage variation on a distribution system can easily be described through worst case scenario. This can be done by using the simple algebraic equation (6). If we consider the worst case scenario as minimum load and maximum generation, then we can write:

$$
P_{L}=0, \quad Q_{L}=0, \quad \text { and } \quad P_{G}=P_{G \max }
$$

Again, if we assume that the system is operating at unity power factor, then $\pm Q_{G}$ and $\pm Q_{C}$ will be zero. In this condition equation (6) can be written as:

$$
\Delta V_{\text {worst }}=V_{G E N \max }-V_{S} \approx R P_{G \max }
$$

For large system, the worst case voltage variation is

$$
\Delta V_{\text {worstji }}=V_{\text {jmax }}-V_{i} \approx R_{i j} P_{G j \max }
$$

Now, if the system is operated at a power factor other than unity, i.e., $\pm Q_{G} \neq 0$ and $\pm Q_{C} \neq 0$, then under worst case scenario by using equation (6) we can write

$$
\Delta V_{\text {worst }} \approx R P_{G \max }+X\left( \pm Q_{G \max } \pm Q_{C}\right)
$$

and for large systems

$$
\Delta V_{\text {worstji }} \approx R_{i j} P_{G \operatorname{maxj}}+X_{i j}\left( \pm Q_{G \operatorname{maxj}} \pm Q_{C j}\right)
$$

From equation (11) it is clear that the voltage variation will reduce if the second term of right side has some smaller or negative values which generally depend on the imported or exported reactive power. Therefore, voltage of a distribution network with DG can be controlled by using reactive power compensation.

\section{Voltage Control using Reactive Power COMPENSATION}

As discussed previously, DG may export or import power to the grid. When DG exports power, then equation (11) can be written as

$$
\Delta V_{\text {worst }} \approx R P_{G \max }+X\left(+Q_{G \max } \pm Q_{C}\right)
$$




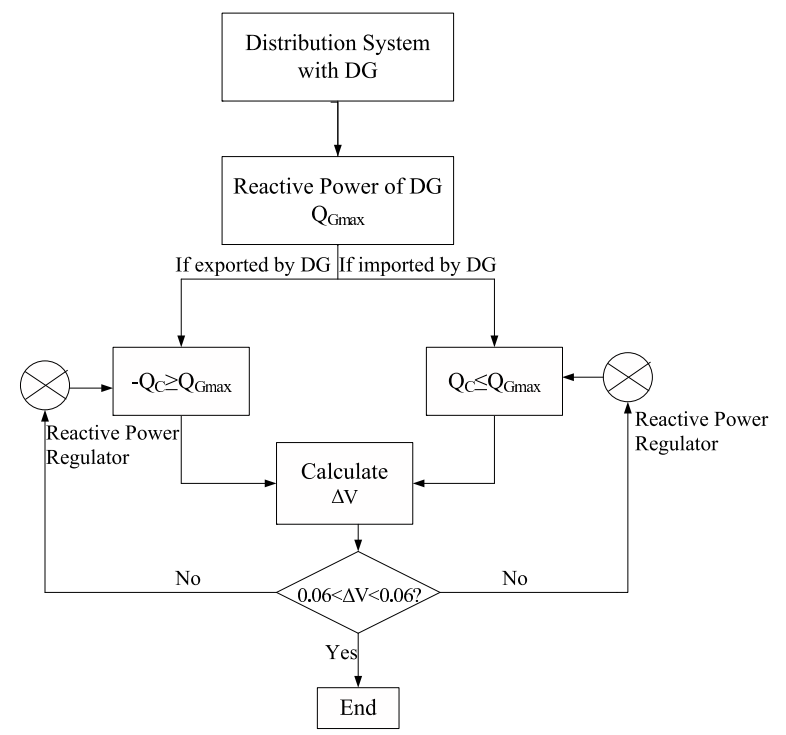

Fig. 5. Voltage control algorithm under worst case scenario

and that of for large systems

$$
\Delta V_{\text {worstji }} \approx R_{i j} P_{G \operatorname{maxj}}+X_{i j}\left(+Q_{G \operatorname{maxj}} \pm Q_{C j}\right)
$$

From equation (12), it is seen that to reduce the second part, the compensator must import reactive power which should equal to or greater than that of the reactive power exported by the generator.

Again, if DG imports reactive power, equation (11) can be written as

$$
\Delta V_{\text {worst }} \approx R P_{G \max }+X\left(-Q_{G \max } \pm Q_{C}\right)
$$

and for large systems it can be written as

$$
\Delta V_{\text {worstji }} \approx R_{i j} P_{G \operatorname{maxj}}+X_{i j}\left(-Q_{G \operatorname{maxj}} \pm Q_{C j}\right)
$$

From equation (13), it is clear that there is a possibility of being second term negative or zero when the compensator exports reactive power less than or equal to the imported reactive power of DG. Under these situation, i.e., under the consideration of worst case scenario of the network, the control algorithm is shown in Fig. 5.

Therefore, from analysis of worst case scenario it is clear that reactive power compensation is very essential in order to keep the voltage profile within the specified limits. The reactive power compensation can be provided by using differen types of FACTS devices. Since the target of this work is to do some static analysis to improve the voltage profile of distribution networks with DG, the shunt capacitors are used as compensators. Some simulation results are shown in the following section.

\section{Simulation Results}

The single line diagram of a 15-bus Kumamoto, Japanese system is shown in Fig. 6. The detailed of this model can be found in [18], [19] At first we have considered that bus-1 is connected to the grid and there is no distributed generation within the system. Under this condition the voltages at different nodes are shown in Fig. 7.

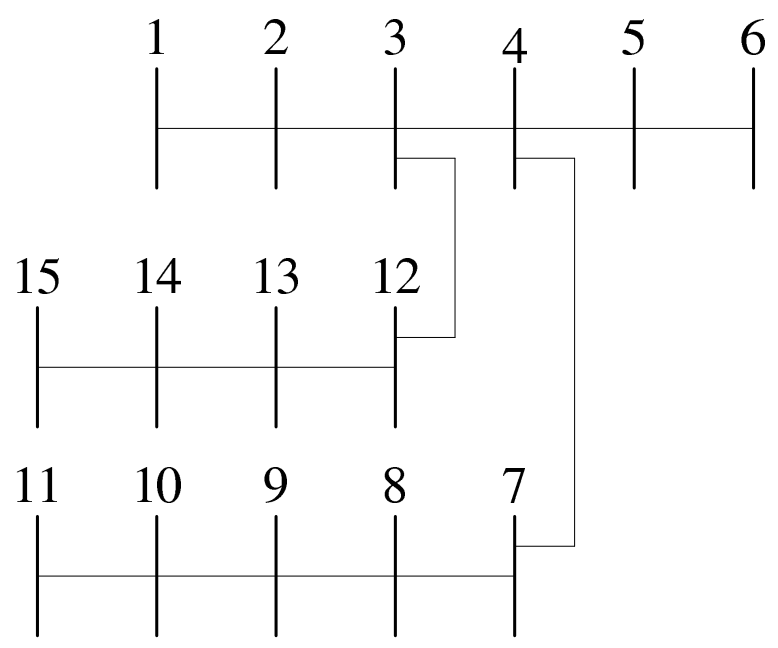

Fig. 6. 15-bus Kumamoto, Japanese system

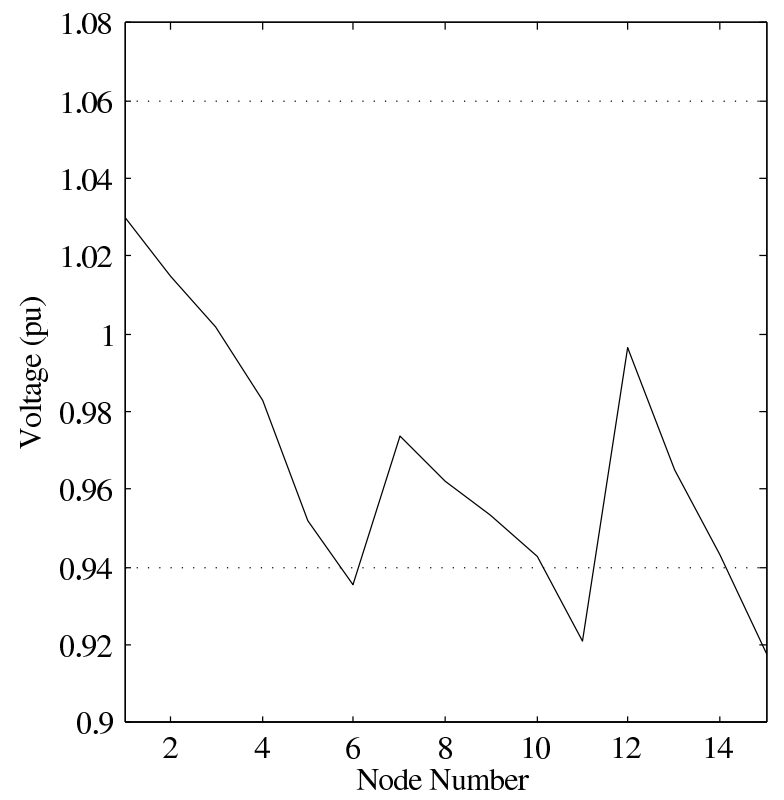

Fig. 7. Voltage profile without DG

From Fig. 7, it is seen that the voltages at nodes 6, 15, and 11 are below the specified limits where the specified limits are considered as $\pm 6 \%$ because these nodes are far away from the main grid supply point. Now, if a synchronous generator is connected at bus-4, a photovoltaic generator is connected at bus-13, and a wind generator is connected at bus-10, the voltages at different nodes are shown in Fig. 8.

Fig. 8 clearly indicates that there are voltage rises at bus4 and bus-13 as these distributed generators export reactive power. But there is voltage drop at bus-10. This is due to the absorbtion of reactive power by induction generator which is used in wind generation. Therefore, reactive power compensation is necessary. The voltage profile of the considered distribution system with DG and reactive power compensation is shown in Fig. 9.

Fig. 9 shows the improved voltage profile as compared to Fig. 8. The amount of compensated reactive power can easily be calculated from voltage control algorithm as shown 


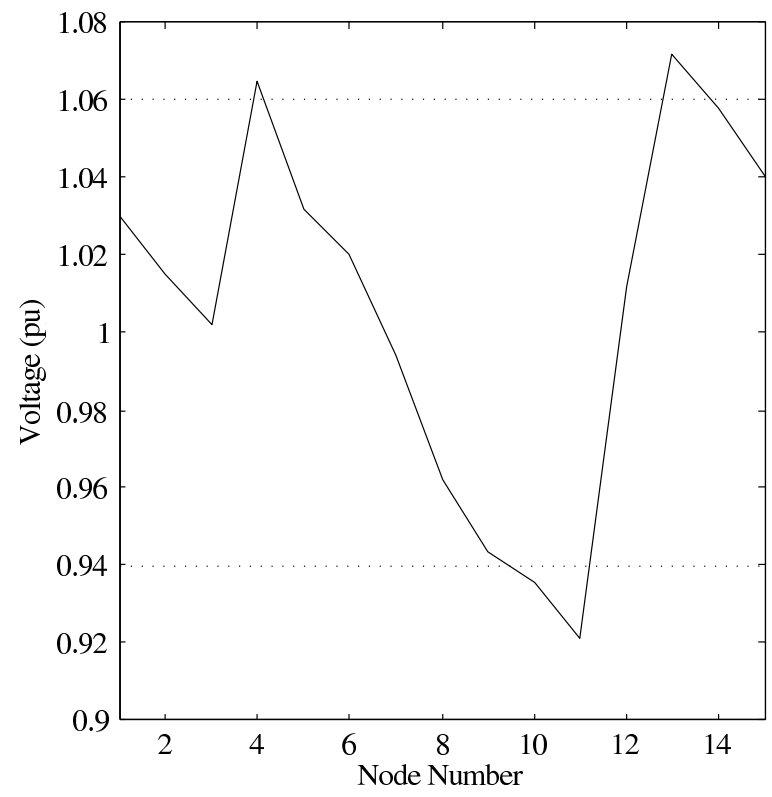

Fig. 8. Voltage profile with DG

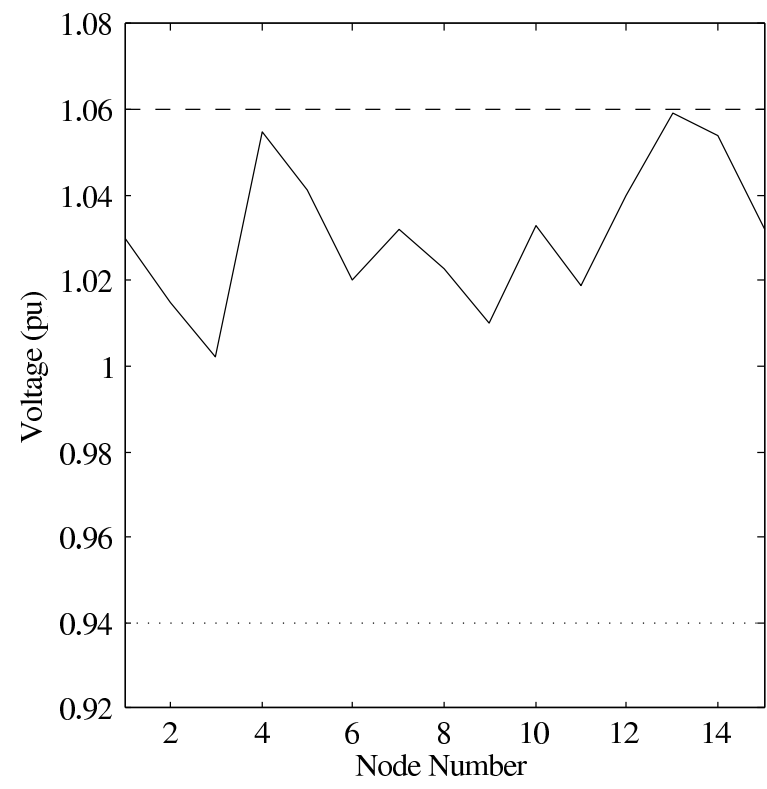

Fig. 9. Voltage profile with DG and reactive power compensation

in Fig. 5. Therefore, reactive power compensation is useful to maintain the voltage profile of a distribution network with DG within the specified limits.

\section{CONCLUSION}

This paper presents an idea about the voltage variation within a distribution network without DG and with DG. From the the derived voltage variation formula, the worst case scenario of the network is considered as it is very useful for both the customers and DNSPs. The derived voltage variation formulae determine the voltage profile of small as well as large distribution network. Based on the worst case scenario of distribution network, a voltage control approach is proposed through reactive power compensation. Finally, a static analysis of voltage control using reactive power compensation is presented to maintain the specified voltage profile of distribution networks. Simulation results clearly show that specified voltage can be obtained by using reactive power compensation. Future works will deal with the design of a voltage controller based on same algorithm by considering the dynamics of the systems.

\section{REFERENCES}

[1] P. Chiradeja and R. Ramakumar, "An approach to quantify the technical benefits of distributed generation," IEEE Trans. on Energy Conversion, vol. 19, no. 4, pp. 764-773, 2004.

[2] L. F. Ochoa, A. Padilha-Feltrin, and G. P. Harrison, "Evaluating distributed generation impacts with a multiobjective index," IEEE Trans. on Power Delivery, vol. 21, no. 3, pp. 1452-1458, 2006.

[3] C. L. Masters, "Voltage rise: the big issue when connecting embedded generation to long $11 \mathrm{kV}$ overhead lines," IET Power Engineering Journal, vol. 16, no. 2, pp. 5-12, 2002.

[4] L. F. Ochoa, "Time-series based maximization of distributed wind power generation integration," IEEE Trans. on Energy Conversion, vol. 23 , no. 3, pp. 968-974, 2008

[5] V. H. M. Quezada, J. R. Abbad, and T. G. S. Romn, "Assessment of energy distribution losses for increasing penetration of distributed generation," IEEE Trans. on Power Systems, vol. 21, no. 2, pp. 533540, 2006.

[6] C. Wang and M. H. Nehrir, "Analytical approaches for optimal placement of distributed generation sources in power systems," IEEE Trans. on Power Systems, vol. 19, no. 4, pp. 2068-2076, 2004.

[7] N. Acharya, P. Mahat, and N. Mithulananthan, "An analytical approach for dg allocation in primary distribution network," Int. J. Elect. Power and Energy Syst., vol. 28, no. 10, pp. 669-678, 2006.

[8] C. J. Dent, L. F. Ochoa, and G. P. Harrison, "Network distribution capacity analysis using OPF with voltage step constraints," IEEE Trans. on Power Systems, vol. 25, no. 1, pp. 296-304, 2010.

[9] L. F. Ochoa, C. J. Dent, and G. P. Harrison, "Distribution network capacity assessment: Variable DG and active networks," IEEE Trans. on Power Systems, vol. 25, no. 1, pp. 87-95, 2010.

[10] M. A. Mahmud, M. J. Hossain, and H. R. Pota, "Worst case scenario for large distribution networks with distributed generation," Accepted for Publication in IEEE PES General Meeting, 24-28 July, 2011.

[11] _ _Analysis of voltage rise effects on distribution systems with distributed generation," Accepted for Publication in IFAC World Congress, 28 Aug-2 Sep, 2011

[12] C.-L. Su, "Stochastic evaluation of of voltages in distribution network with distributed generation using detailed distribution models," IEEE Trans. on Power Systems, vol. 25, no. 2, pp. 786-795, 2010.

[13] R.-H. Liang and Y.-S. Wang, "Fuzzy-based reactive power and voltage control in a distribution system," IEEE Trans. on Power Delivery, vol. 18, no. 2, pp. 610-618, 2003.

[14] M. B. Liu, C. A. Caizares, and W. Huang, "Reactive power and voltage control in distribution systems with limited switching operations," IEEE Trans. on Power Systems, vol. 24, no. 2, pp. 889-899, 2009.

[15] A. Keane, L. F. Ochoa, E. Vittal, C. J. Dent, and G. P. Harrison, "Enhanced utilization of voltage control resources with distributed generation," IEEE Trans. on Power Systems, vol. 26, no. 1, pp. 252 260, 2011.

[16] P. M. S. Carvalho, P. F. Correia, and L. A. F. M. Ferreira, "Distributed reactive power generation control for voltage rise mitigation in distribution networks," IEEE Trans. on Power Systems, vol. 23, no. 2, pp. 766-772, 2008.

[17] W. H. Kersting, Distribution System Modeling and Analysis. London: CRC Press, Second Edition, 2007.

[18] N. K. Roy, M. A. Mahmud, M. J. Hossain, and H. R. Pota, "Impact of distributed wind generation on distribution networks," In Proc. of Solar2010, the 48th AuSES Annual Conference, 1-3 Dec, 2010.

[19] F. M. Gonzlez-Longatt, "Impact of distributed generation over power losses on distribution system," In Proc. of 9th International Conference on Electrical Power Quality and Utilisation, 9-11 Oct, 2007. 